\title{
Validação de equações antropométricas específicas e generalizadas para estimativa do percentual de gordura corporal em estudantes de Educação Física do sexo masculino
}

CDD. 20.ed. 796.022

796.033

http://dx.doi.org/10.1590/1807-55092015000100013
Diego Rodrigo BOTH*

Silvana Corrêa MATHEUS*

Mauri Schwanck BEHENCK*
*Centro de Educação Física e Desportos, Universidade Federal de Santa Maria.

\section{Resumo}

0 objetivo deste estudo foi verificar a validade das equações específicas de GuEDES e GuEDEs ${ }^{7}$ e das generalizadas de Petroski ${ }^{9}$ em universitários do sexo masculino. A amostra foi constituída de 104 universitários de Educação Física com idade média de $21,7 \pm 2,7$ anos, submetidos à pesagem hidrostática (PH) e à antropometria (dobras cutâneas e perímetros corporais). Não foram observadas diferenças estatisticamente significativas entre as medidas de percentual de gordura pela PH e nove equações de PEtroski ${ }^{9}$. Todos os resultados obtidos pelas equações de GUEDES e GUEDES ${ }^{7}$ apresentaram diferenças significativas quando comparados com os da PH. Entre as equações analisadas, oito desenvolvidas por Petroskl ${ }^{9}$ apresentaram validade concorrente na amostra estudada.

Palavras-Chave: Composição corporal; Universitários; Pesagem hidrostática; Antropometria.

\section{Introdução}

No cenário em que vivemos, a composiçáo corporal tem ganhado cada vez mais importância, isso se deve a sua íntima ligação com a saúde humana. $\mathrm{O}$ acúmulo de adiposidade pode proporcionar o surgimento de doenças crônicas náo transmissíveis, principalmente as de cunho cardiovascular ${ }^{1}$.

A gordura corporal é um dos elementos quantificáveis da composição corporal. Tal variável pode ser estimada por meio de métodos laboratoriais, como a pesagem hidrostática $(\mathrm{PH})$, a absortometria radiológica de dupla energia (DXA), a ressonância magnética, a diluição de óxido de deutério, a excreção de creatinina urinária, entre outros ${ }^{2}$. A gordura corporal também pode ser avaliada por métodos clínicos, tais como, a antropometria e a impedância bioelétrica ${ }^{3}$. Dentre os métodos laboratoriais, a PH merece destaque especial, pois sua medida é considerada de grande precisão e normalmente é a técnica referência utilizada no desenvolvimento de novos instrumentos de quantificação da gordura corporal ${ }^{4}$.

Em relação aos métodos clínicos, a antropometria é o mais difundido em termos de avaliação do percentual de gordura corporal (\%GC), pois utiliza equipamentos de baixo custo, promove facilidade e rapidez na coleta dos dados e, além disso, não é uma técnica invasiva ${ }^{5}$. $\mathrm{Na}$ literatura pode-se identificar uma infinidade de estudos que desenvolveram equaçóes antropométricas de predição da gordura corporal aplicáveis a diversos segmentos populacionais, como crianças e adolescentes ${ }^{6}$, universitários ${ }^{7}$, adultos ${ }^{8-9}$, idosos ${ }^{10-11}$, militares ${ }^{12}$, entre outros.

Entre os estudos nacionais, destacam-se os de Guedes e Guedes ${ }^{7}$ e Petroski ${ }^{9}$, que desenvolveram modelos matemáticos preditivos da densidade corporal, nos quais foram empregadas variáveis antropométricas provenientes de pessoas da regiáo Sul do Brasil. Guedes e Guedes ${ }^{7}$ utilizaram universitários da Universidade Federal de Santa Maria com idade entre 18 e 35 anos no desenvolvimento das equaçôes, desse modo são denominadas como equações específicas. Já as desenvolvidas por PETROSKI ${ }^{9}$ tiveram origem a partir de dados de indivíduos das regióes central do Rio Grande do Sul e litorânea de Santa Catarina, abrangendo uma ampla faixa etária (18 a 66 anos), caracterizando-as como equações generalizadas. 
No entanto, estes estudos ${ }^{7,9}$, bem com estudos posteriores de validação cruzada destas equações ${ }^{13-15}$, utilizaram, entre outras análises estatísticas, o coeficiente de correlação ao comparar os modelos matemáticos que estimam a densidade corporal para verificar o grau de concordância entre os resultados obtidos. Entretanto, algumas críticas têm sido levantadas com relação à utilização do coeficiente de correlação como critério de análise da validaçáo cruzada para comparação de duas técnicas de medida. Bland e ALtMan ${ }^{16}$ destacam que o coeficiente de correlação mede a força de relação entre duas variáveis e não o acordo entre elas. Também, enfatizam que a mudança na escala de medida náo afeta a correlaçáo, mas certamente afeta a concordância entre as medidas; além disso, a correlação depende do número de observações. E ainda, dados podem apresentar pouca concordância e ao mesmo tempo podem revelar elevada correlação. Sendo assim, a correlação poderia superestimar a associação, implicando conclusões errôneas quanto à validade do instrumento ${ }^{17}$.

$\mathrm{Na}$ tentativa de obter um método mais confiável de verificação de concordância, Bland e Altman ${ }^{16}$

\section{Método}

Trata-se de um estudo transversal, no qual a amostra foi constituída a partir de um universo de 268 alunos, do sexo masculino que cursavam graduação em Educação Física na Universidade Federal de Santa Maria - UFSM, localizada na cidade de Santa Maria, região central do Rio Grande do Sul/Brasil. Utilizouse um cálculo amostral que se baseou na média e desvio-padrão do \%GC (determinado a partir de um estudo piloto com 20 indivíduos) e Intervalo de Confiança (IC) estabelecido em 95\%. O cálculo indicou 92 indivíduos necessários para representar o total de alunos. No entanto, foi avaliada uma amostra de 104 universitários voluntários, que atenderam os seguintes critérios de inclusão: possuir idade entre 18 e 30 anos, não apresentar dificuldade na exalação do ar durante a pesagem subaquática, não possuir deficiência que pudesse interferir na composição corporal e se autodeclarar branco (cor/etnia).

Todos os participantes deste estudo foram informados dos procedimentos aos quais seriam submetidos e assinaram o termo de consentimento livre e esclarecido.

Este estudo é parte de uma pesquisa em desenvolvimento a qual possui aprovaçáo do Comitê de Ética da Universidade Federal de Santa Maria propuseram a análise dos escores residuais. É um método aplicado para avaliar a concordância absoluta entre dois valores expressos na mesma unidade de medida. Consiste em plotar a diferença entre a medida de determinada variável mensurada a partir de um critério de referência e aquela produzida pelo instrumento de avaliaçáo, contra a média dessas duas medidas. Ou seja, baseia-se na construção de um gráfico cartesiano de dispersão cujas médias e diferenças entre os métodos encontram-se no eixo das abscissas e das ordenadas, respectivamente. Permite avaliar a magnitude das diferenças entre dois procedimentos de medida de uma mesma variável ${ }^{18}$. Além disso, este método permite verificar a presença do viés de medi$\mathrm{da}$, ou seja, o quanto a diferença entre os resultados das medidas se distancia de zero. Essa técnica tem se mostrado altamente informativa e requer pouca sofisticação nas rotinas computacionais ${ }^{19}$.

Com base nas informaçôes apresentadas, o objetivo deste estudo é verificar a validade cruzada das equaçôes específicas de Guedes e Guedes ${ }^{7}$ e das generalizadas de Petroski ${ }^{9}$ em universitários do sexo masculino.

(CAAE: 11511112.8.0000.5346), com base na Resolução 196/96 do Conselho Nacional de Saúde para Pesquisas em Seres Humanos.

\section{Antropometria}

A massa corporal foi determinada com uma balança digital da marca Marte ${ }^{\circ}$, com resolução de $0,1 \mathrm{~kg}$, e capacidade máxima de $180 \mathrm{~kg}$. A estatura foi verificada utilizando-se um estadiômetro fixo Cardiomed ${ }^{\circ}$, com resolução de $0,1 \mathrm{~cm}$ e extensão máxima de 220 $\mathrm{cm}$. Para ambos os procedimentos foram utilizadas as padronizações sugeridas por STEWART et al. ${ }^{20}$.

Foram mensuradas dobras cutâneas em 10 pontos anatômicos seguindo três protocolos diferentes: subescapular, tríceps, bíceps, supra-ilíaca, abdominal, coxa média, panturrilha média ${ }^{20}$; peitoral, axilar média ${ }^{21} ; \mathrm{e}$ coxa terço superior ${ }^{7}$. Foi utilizado um adipômetro da marca Cescorf ${ }^{\natural}$, com resolução de $0,1 \mathrm{~mm}$.

Os perímetros corporais foram mensurados nos seguintes locais: antebraço e abdômen ${ }^{21}$. Utilizou-se uma fita antropométrica inextensível da marca Cescorf ${ }^{\oplus}$, com resolução de $0,1 \mathrm{~cm}$ e extensão máxima de $200 \mathrm{~cm}$. 
Um único avaliador treinado e com experiência em antropometria realizou todas as medidas, as quais foram mensuradas duas vezes de forma rotacional (não consecutiva) no lado direito do corpo, obtendo-se um erro maior que $5 \%$ entre as medidas de dobras cutâneas e de $1 \%$ para os perímetros corporais, realizou-se uma terceira, definindo-se a média de dois valores mais próximos como a medida. $\mathrm{O}$ erro de medida apresentado pelo avaliador foi de 2,0\% para as medidas de dobras cutâneas e de $0,3 \%$ para os perímetros corporais.

\section{Pesagem hidrostática (H)}

Utilizou-se um tanque projetado e apropriado para a $\mathrm{PH}$, com aproximadamente $170 \mathrm{~cm}$ de diâmetro e $150 \mathrm{~cm}$ de profundidade. Sobre o tanque, está afixada uma balança da marca Filizola ${ }^{\circledR}$, com resolução de $0,01 \mathrm{~kg}$ e capacidade máxima de $6 \mathrm{~kg}$, a qual foi empregada para verificar o peso subaquático. Os avaliados posicionavam-se sentados em uma cadeira confeccionada com tubos de policloreto de vinila (PVC), a qual está acoplada por meio de correntes à balança. Para a pesagem subaquática, o sujeito inclinava-se para a frente, aproximando o tórax das coxas, submergindo totalmente. A seguir, e este foi estimulado a eliminar, através de uma expiração forçada, a maior quantidade possível do ar mantido nos pulmóes. Foram realizadas de sete a 12 tentativas, e registrado como peso subaquático bruto a média dos três maiores valores com diferença $\leq 100 \mathrm{~g}$. Os procedimentos utilizados para a verificação do peso subaquático foram os descritos por HEYWARD ${ }^{22}$. A temperatura da água foi mantida entre $32^{\circ}$ e $36^{\circ} \mathrm{C}$ para as avaliaçóes, medidas com um termômetro (Incoterm ${ }^{\circledR}$ ).

O volume residual foi estimado através da equação de Goldman e Becklake ${ }^{23}$.

Após a mensuração do peso subaquático e o volume residual, os valores foram aplicados na seguinte equação de determinação da densidade corporal (Dc):

$$
\operatorname{Dc}\left(\frac{\mathrm{g}}{\mathrm{cm}^{3}}\right)=\frac{\mathrm{MC}}{\left(\frac{\mathrm{MC}-\mathrm{Ps}}{\mathrm{Da}}\right)-(\mathrm{VR}+0,1)}
$$

Onde:

Dc = densidade corporal;

$\mathrm{MC}=$ massa corporal;

Ps = peso subaquático;

$\mathrm{Da}=$ densidade da água;

$\mathrm{VR}$ = volume residual;

0,1 = constante do gás gastrointestinal.

Todas as avaliações foram realizadas no Laboratório de Cineantropometria por um único avaliador.

A TABELA 1 apresenta as equaçóes específicas e generalizadas de estimativa da Dc.

Para a determinaçáo do \%GC a partir da Dc foram utilizadas as equaçóes desenvolvidas para populaçóes específicas, que levam em consideração a etnia, a idade e o $\operatorname{sexo}^{24}$.

TABELA 1 - Equações específicas e generalizadas de estimativa da Dc em homens.

\begin{tabular}{|c|c|c|c|c|}
\hline \multicolumn{2}{|r|}{ Equaçóes } & \multirow[t]{2}{*}{$\mathbf{r}$} & \multirow[t]{2}{*}{$\mathbf{r}^{2}$} & \multirow[t]{2}{*}{ EPE } \\
\hline & Específicas (GUEDES e GUEDES'7) & & & \\
\hline Eq. GM1 & $\mathrm{Dc}=1,13060-0,05437(\log 10 \mathrm{DCAB})$ & 0,864 & $\mathrm{NI}$ & 0,0064 \\
\hline Eq. GM2 & $\mathrm{Dc}=1,15929-0,06550(\log 10 \mathrm{DCAB}+\mathrm{DCTR})$ & 0,880 & $\mathrm{NI}$ & 0,0061 \\
\hline Eq. GM3 & $\mathrm{Dc}=1,17136-0,06706(\log 10 \mathrm{DCAB}+\mathrm{DCTR}+\mathrm{DCSI})$ & 0,894 & $\mathrm{NI}$ & 0,0057 \\
\hline Eq. GM4 & $\mathrm{Dc}=1,18282-0,07030(\log 10 \mathrm{DCAB}+\mathrm{DCTR}+\mathrm{DCSI}+\mathrm{DCAM})$ & 0,894 & $\mathrm{NI}$ & 0,0057 \\
\hline Eq. GM5 & $\mathrm{Dc}=1,20436-0,07848(\log 10 \mathrm{DCAB}+\mathrm{DCTR}+\mathrm{DCSI}+\mathrm{DCAM}+\mathrm{DCSB})$ & 0,894 & NI & 0,0057 \\
\hline Eq. GM6 & $\mathrm{Dc}=1,21546-0,08119(\log 10 \mathrm{DCAB}+\mathrm{DCTR}+\mathrm{DCSI}+\mathrm{DCAM}+\mathrm{DCSB}+\mathrm{DCCS})$ & 0,889 & $\mathrm{NI}$ & 0,0056 \\
\hline Eq. GM7 & $\begin{array}{l}\mathrm{Dc}=1,22098-0,08214(\log 10 \mathrm{DCAB}+\mathrm{DCTR}+\mathrm{DCSI}+\mathrm{DCAM}+\mathrm{DCSB}+\mathrm{DCCS}+\mathrm{D} \\
\mathrm{CPM})\end{array}$ & 0,904 & $\mathrm{NI}$ & 0,0054 \\
\hline Eq. GM8 & $\begin{array}{l}\mathrm{Dc}=1,22627-0,08384(\log 10 \mathrm{DCAB}+\mathrm{DCTR}+\mathrm{DCSI}+\mathrm{DCAM}+\mathrm{DCSB}+\mathrm{DCCS}+\mathrm{D} \\
\mathrm{CPM}+\mathrm{DCBI})\end{array}$ & 0,901 & $\mathrm{NI}$ & 0,0055 \\
\hline
\end{tabular}


TABELA 1 - Equações específicas e generalizadas de estimativa da Dc em homens (continuação).

\begin{tabular}{|c|c|c|c|c|}
\hline & Equaçóes & $\mathbf{r}$ & $\mathbf{r}^{2}$ & EPE \\
\hline & \multicolumn{4}{|l|}{ Generalizadas (PETROSKI ${ }^{9}$ ) } \\
\hline Eq. PM1 & $\begin{array}{l}\text { Dc }=1,10194032-0,00031836(\text { DCSB, DCTR, DCBI, DCPT, DCAM, DCSI, } \\
\text { DCAB, DCCX, DCPM) + 0,00000029(DCSB, DCTR, DCBI, DCPT, DCAM, } \\
\text { DCSI, DCAB, DCCX, DCPM) } 2 \text { - 0,00029542(IDADE) }\end{array}$ & 0,887 & 0,786 & 0,0072 \\
\hline Eq. PM2 & $\begin{array}{l}\text { Dc }=1,08516305-0,00028465(\text { DCSB, DCTR, DCBI, DCPT, DCAM, DCSI, } \\
\text { DCAB, DCCX, DCPM }+0,00000026(\text { DCSB, DCTR, DCBI, DCPT, DCAM, } \\
\text { DCSI, DCAB, DCCX, DCPM } 2-0,00021018(\text { IDADE })+0,00173856(\text { PAT })- \\
0,00043254(\text { PAB })\end{array}$ & 0,894 & 0,800 & 0,0070 \\
\hline Eq. PM3 & $\begin{array}{l}\text { Dc }=1,10038145-0,00035804(\text { DCSB, DCTR, DCPT, DCAM, DCSI, DCAB, } \\
\text { DCCX }+0,00000036(\text { DCSB, DCTR, DCPT, DCAM, DCSI, DCAB, DCCX }) 2- \\
0,00025154(\text { IDADE }) .\end{array}$ & 0,880 & 0,780 & 0,0073 \\
\hline Eq. PM4 & $\begin{array}{l}\mathrm{Dc}=1,08566598-0,00032750(\mathrm{DCSB}, \mathrm{DCTR}, \mathrm{DCPT}, \mathrm{DCAM}, \mathrm{DCSI}, \mathrm{DCAB}, \\
\mathrm{DCCX})+0,00000036(\mathrm{DCSB}, \mathrm{DCTR}, \mathrm{DCPT}, \mathrm{DCAM}, \mathrm{DCSI}, \mathrm{DCAB}, \mathrm{DCCX}) 2 \\
-0,00017521(\mathrm{IDADE})+0,00161816(\mathrm{PAT})-0,00041043(\mathrm{PAB})\end{array}$ & 0,892 & 0,795 & 0,0071 \\
\hline Eq. PM5 & $\begin{array}{l}\text { Dc }=1,09995680-0,00055475(\text { DCSB, DCTR, DCBI, DCPT, DCAM, DCSI }+ \\
0,00000107(\text { DCSB, DCTR, DCBI, DCPT, DCAM, DCSI ) } 2-0,00023367(\text { IDADE). }\end{array}$ & 0,881 & 0,776 & 0,0074 \\
\hline Eq. PM6 & $\begin{array}{l}\mathrm{Dc}=1,08555470-0,00050212(\mathrm{DCSB}, \mathrm{DCTR}, \mathrm{DCBI}, \mathrm{DCPT}, \mathrm{DCAM}, \mathrm{DCSI})+ \\
0,00000104(\mathrm{DCSB}, \mathrm{DCTR}, \mathrm{DCBI}, \mathrm{DCPT}, \mathrm{DCAM}, \mathrm{DCSI}) 2-0,00015217(\mathrm{IDA}- \\
\mathrm{DE})+0,00169842(\mathrm{PAT})-0,00044620(\mathrm{PAB})\end{array}$ & 0,889 & 0,790 & 0,0071 \\
\hline Eq. PM7 & $\begin{array}{l}\text { Dc }=1,10726863-0,00081201(\mathrm{DCSB}, \mathrm{DCTR}, \mathrm{DCSI}, \mathrm{DCPM})+ \\
0,00000212(\mathrm{DCSB}, \mathrm{DCTR}, \mathrm{DCSI}, \mathrm{DCPM}) 2-0,00041761(\mathrm{IDADE}) .\end{array}$ & 0,875 & 0,765 & 0,0075 \\
\hline Eq. PM8 & $\begin{array}{l}\text { Dc }=1,09255357-0,00067980(\text { DCSB, DCTR, DCSI, DCPM })+ \\
0,00000182(\text { DCSB, DCTR, DCSI, DCPM }) 2-0,00027287(\text { IDADE })+ \\
0,00204435(\text { PAT })-0,00060405(P A B) .\end{array}$ & 0,889 & 0,791 & 0,0071 \\
\hline Eq. PM9 & $\begin{array}{l}\text { Dc }=1,10539106-0,00089839(\text { DCSB, DCTR, DCBI, DCSI })+ \\
0,00000278(\text { DCSB, DCTR, DCBI, DCSI }) 2-0,00035250(\text { IDADE }) .\end{array}$ & 0,874 & 0,764 & 0,0075 \\
\hline Eq. PM10 & $\begin{array}{l}\text { Dc }=1,09158117-0,00077719(\mathrm{DCSB}, \mathrm{DCTR}, \mathrm{DCBI}, \mathrm{DCSI})+ \\
0,00000257(\mathrm{DCSB}, \mathrm{DCTR}, \mathrm{DCBI}, \mathrm{DCSI}) 2-0,00022634(\mathrm{IDADE})+ \\
0,00195027(\mathrm{PAT})-0,00057011(\mathrm{PAB}) .\end{array}$ & 0,887 & 0,786 & 0,0072 \\
\hline Eq. PM11 & $\begin{array}{l}\mathrm{Dc}=1,10491700-0,00099061(\mathrm{DCSB}, \mathrm{DCTR}, \mathrm{DCSI})+0,00000327(\mathrm{DCSB}, \\
\text { DCTR, DCSI)2- 0,00034527(IDADE). }\end{array}$ & 0,871 & 0,759 & 0,0076 \\
\hline Eq. PM12 & $\begin{array}{l}\mathrm{Dc}=1,09360757-0,00086876(\mathrm{DCSB}, \mathrm{DCTR}, \mathrm{DCSI})+0,00000327(\mathrm{DCSB} \\
\text { DCTR, DCSI } 2-0,00021422(\mathrm{IDADE})+0,00191721(\mathrm{PAT})-0,00059091(\mathrm{PAB})\end{array}$ & 0,884 & 0,781 & 0,0072 \\
\hline Eq. PM13 & $\begin{array}{l}\mathrm{Dc}=1,10404686-0,00111938(\mathrm{DCSB}, \mathrm{DCTR}, \mathrm{DCPT})+0,00000391(\mathrm{DCSB}, \\
\text { DCTR, DCPT) } 2-0,00027884(\mathrm{IDADE})\end{array}$ & 0,873 & 0,763 & 0,0075 \\
\hline Eq. PM14 & $\begin{array}{l}\mathrm{Dc}=1,08974189-0,00098446(\mathrm{DCSB}, \mathrm{DCTR}, \mathrm{DCPT})+0,00000376(\mathrm{DCSB} \\
\mathrm{DCTR}, \mathrm{DCPT}) 2-0,00017218(\mathrm{IDADE})+0,00191020(\mathrm{PAT})-0,00054056(\mathrm{PAB})\end{array}$ & 0,885 & 0,783 & 0,0072 \\
\hline Eq. PM15 & $\begin{array}{l}\text { Dc }=1,10098229-0,00145899(\text { DCTR, DCAM })+0,00000701(\text { DCTR, DCAM }) 2 \\
-0,00032770(\text { IDADE }) .\end{array}$ & 0,885 & 0,784 & 0,0072 \\
\hline Eq. PM16 & $\begin{array}{l}\mathrm{Dc}=1,08843264-0,00130623(\mathrm{DCTR}, \mathrm{DCAM})+0,00000710(\mathrm{DCTR}, \\
\text { DCAM }) 2-0,00021414(\mathrm{IDADE})+0,00182587(\mathrm{PAT})-0,00052569(\mathrm{PAB}) .\end{array}$ & 0,896 & 0,803 & 0,0070 \\
\hline
\end{tabular}

\section{Análise estatística}

Para a análise dos dados utilizou-se dois programas: o Statistical Package for the Social Sciences (SPSS) versão 14.0 e o MedCalc 12 (construção dos gráficos). A normalidade dos dados foi testada pelo teste de Kolmogorov-Smirnov. No processo de validação cruzada das equaçóes antropométricas, foram comparadas as médias por meio do teste " $\mathrm{t}$ " para amostras dependentes, correlação linear de Pearson $(\mathrm{r})$ e coeficiente de determinação $\left(\mathrm{r}^{2}\right)$, também foram analisados o erro constante (EC), o erro total (ET) e o erro padrão de estimativa (EPE) de acordo com os critérios sugeridos por LoHMAN ${ }^{25}: r \geq 0,79$; $p$ $>0,05$; e EPE $\leq 3,5 \%$. Ainda foi realizada a análise da dispersão dos resíduos pelo método proposto por Bland e Altman ${ }^{16}$. O nível de significância adotado foi de $5 \%$. 


\section{Resultados}

A TABELA 2 apresenta os resultados das características descritivas da amostra estudada com valores de média, desvio-padrão e escores mínimos e máximos das variáveis antropométricas.

São apresentados na TABELA 3 os resultados de validaçấo cruzada das equaçôes específicas e generalizadas para estimativa do \%GC. Para a comparaçáo dos resultados obtidos entre os métodos de avaliaçáo do \%GC, utilizou-se os dados fornecidos pela $\mathrm{PH}$ como valores de referência. Apesar de todas as equações utilizadas neste estudo fornecerem valores de Dc, optou-se por converter tais valores em gordura corporal relativa por meio das equaçóes específicas propostas por HeYward e WaGNeR ${ }^{24}$.

Com relação à associação, o valor do coeficiente de correlaçáo obtido entre os resultados da $\mathrm{PH}$ e das equaçôes antropométricas variou de 0,77 a 0,83 para as de Guedes e Guedes ${ }^{7}$ e de 0,78 a 0,84 para as de Petroski ${ }^{9}$. Já o coeficiente de determinação $\left(\mathrm{r}^{2}\right)$ para as equaçóes de Guedes e Guedes ${ }^{7}$ variou de 0,59 a 0,69 e para as de Petroski ${ }^{9}$ de 0,61 a 0,70.

Não foram observadas diferenças estatisticamente significativas entre as medidas de \%GC pela $\mathrm{PH}$ e as das equaçóes PM1, PM3, PM4, PM5, PM6, PM7, PM8, PM10 e PM12 de Petroski ${ }^{9}$. No entanto, todos os resultados obtidos pelas equaçóes de Guedes e Guedes ${ }^{7}$ apresentaram diferenças estatisticamente significativas quando comparados com os da PH.

Quanto ao EPE, observa-se que os valores encontrados para as equações de Petroski ${ }^{9}$ variam de $3,1 \%$ a $3,5 \%$, e para as equações de Guedes e Guedes $^{7}$ de 3,3\% a 4,1\%. Também, pode-se perceber que os menores valores, tanto de EC, quanto de ET, foram observados nas equaçóes que não apresentaram diferença significativa $(\mathrm{p}>0,05)$.

TABELA 2 - Características descritivas da amostra.

\begin{tabular}{lcc}
\hline \multirow{2}{*}{ Variáveis } & \multicolumn{2}{c}{ Masculino $(\mathbf{n}=\mathbf{1 0 4})$} \\
\cline { 2 - 3 } & Média $\pm \mathbf{d p}$ & Min. - Max. \\
\hline Idade (anos) & $21,7 \pm 2,7$ & $18-30$ \\
Massa Corporal $(\mathrm{kg})$ & $76,7 \pm 10,8$ & $53,6-105,0$ \\
Estatura $(\mathrm{cm})$ & $177,1 \pm 6,7$ & $161,4-194,0$ \\
IMC $\left(\mathrm{kg} / \mathrm{m}^{2}\right)$ & $24,4 \pm 2,8$ & $17,3-34,0$ \\
Dobras cutâneas (mm) & & \\
Subescapular & $13,0 \pm 5,7$ & $6,3-36,8$ \\
Tríceps & $11,9 \pm 5,4$ & $4,3-31,0$ \\
Bíceps & $5,5 \pm 3,1$ & $2,7-19,0$ \\
Peitoral & $10,3 \pm 6,1$ & $3,1-32,1$ \\
Axilar-média & $12,5 \pm 12,5$ & $4,5-44,6$ \\
Crista-ilíaca & $21,3 \pm 10,5$ & $7,1-54,6$ \\
Abdominal & $24,1 \pm 12,6$ & $6,2-58,3$ \\
Coxa superior & $15,7 \pm 7,6$ & $5,9-40,4$ \\
Coxa média & $14,8 \pm 6,6$ & $5,9-39,9$ \\
Panturrilha medial & $9,8 \pm 4,9$ & $3,7-29,3$ \\
Perímetros (cm) & & \\
Antebraço & $28,0 \pm 1,8$ & $24,0-32,8$ \\
Abdômen & $84,6 \pm 7,7$ & $71,0-109,3$ \\
\hline
\end{tabular}


TABELA 3 - Validação cruzada das equações específicas e generalizadas.

\begin{tabular}{|c|c|c|c|c|c|c|c|c|c|}
\hline \multirow{27}{*}{$\begin{array}{l}\text { PH = pesagem hidros- } \\
\text { tática; } \\
r=\text { correlação de Pearson; } \\
r^{2}=\text { coeficiente de deter- } \\
\text { minação; } \\
p=\text { nível de significância; } \\
\text { EPE = erro padrão de } \\
\text { estimativa; } \\
E C=\text { erro constante; } \\
E T=\text { erro total. }\end{array}$} & Métodos & Média $\pm d p$ & $\mathbf{r}$ & $\mathbf{r}^{2}$ & t & $\mathbf{p}$ & EPE & EC & ET \\
\hline & PH & $16,3 \pm 4,9$ & & & & & & & \\
\hline & Equaçóes & & & & & & & & \\
\hline & Eq. GM1 & $17,8 \pm 5,4$ & 0,77 & 0,59 & $-4,246$ & 0,000 & 3,5 & 1,5 & 14,8 \\
\hline & Eq. GM2 & $17,0 \pm 5,7$ & 0,81 & 0,66 & $-2,127$ & 0,036 & 3,3 & 0,7 & 7,1 \\
\hline & Eq. GM3 & $18,7 \pm 5,9$ & 0,80 & 0,64 & $-6,856$ & 0,000 & 3,8 & 2,4 & 24,3 \\
\hline & Eq. GM4 & $18,7 \pm 6,2$ & 0,79 & 0,62 & $-6,517$ & 0,000 & 3,8 & 2,4 & 24,7 \\
\hline & Eq. GM5 & $18,4 \pm 6,6$ & 0,78 & 0,61 & $-5,312$ & 0,000 & 4,1 & 2,1 & 21,7 \\
\hline & Eq. GM6 & $18,5 \pm 6,7$ & 0,82 & 0,67 & $-5,859$ & 0,000 & 3,8 & 2,2 & 22,8 \\
\hline & Eq. GM7 & $18,5 \pm 6,6$ & 0,83 & 0,69 & $-5,892$ & 0,000 & 3,7 & 2,2 & 22,8 \\
\hline & Eq. GM8 & $18,4 \pm 6,7$ & 0,83 & 0,69 & $-5,659$ & 0,000 & 3,7 & 2,1 & 22,0 \\
\hline & Eq. PM1 & $16,4 \pm 5,7$ & 0,84 & 0,70 & $-0,362$ & 0,718 & 3,1 & 0,1 & 1,1 \\
\hline & Eq. PM2 & $15,6 \pm 5,8$ & 0,83 & 0,69 & 2,319 & 0,022 & 3,2 & $-0,7$ & 7,6 \\
\hline & Eq. PM3 & $16,5 \pm 5,7$ & 0,83 & 0,69 & $-0,708$ & 0,481 & 3,2 & 0,2 & 2,3 \\
\hline & Eq. PM4 & $15,7 \pm 5,8$ & 0,82 & 0,67 & 1,938 & 0,055 & 3,3 & $-0,6$ & 6,4 \\
\hline & Eq. PM5 & $16,8 \pm 5,4$ & 0,79 & 0,62 & $-1,352$ & 0,179 & 3,3 & 0,5 & 4,6 \\
\hline & Eq. PM6 & $15,8 \pm 5,5$ & 0,79 & 0,62 & 1,428 & 0,156 & 3,4 & $-0,5$ & 4,9 \\
\hline & Eq. PM7 & $16,9 \pm 5,5$ & 0,82 & 0,67 & $-1,891$ & 0,061 & 3,2 & 0,6 & 5,9 \\
\hline & Eq. PM8 & $15,8 \pm 5,7$ & 0,82 & 0,67 & 1,603 & 0,112 & 3,3 & $-0,5$ & 5,3 \\
\hline & Eq. PM9 & $17,1 \pm 5,4$ & 0,80 & 0,64 & $-2,290$ & 0,024 & 3,2 & 0,8 & 7,6 \\
\hline & Eq. PM10 & $16,0 \pm 5,6$ & 0,79 & 0,62 & 0,979 & 0,330 & 3,5 & $-0,3$ & 3,4 \\
\hline & Eq. PM11 & $17,2 \pm 5,5$ & 0,79 & 0,62 & $-2,555$ & 0,012 & 3,4 & 0,9 & 8,7 \\
\hline & Eq. PM12 & $16,1 \pm 5,6$ & 0,78 & 0,61 & 0,685 & 0,495 & 3,5 & $-0,2$ & 2,4 \\
\hline & Eq. PM13 & $15,2 \pm 5,3$ & 0,78 & 0,61 & 3,360 & 0,001 & 3,3 & $-1,1$ & 11,5 \\
\hline & Eq. PM14 & $14,4 \pm 5,5$ & 0,78 & 0,61 & 5,558 & 0,000 & 3,4 & $-1,9$ & 19,4 \\
\hline & Eq. PM15 & $15,6 \pm 5,2$ & 0,78 & 0,62 & 2,158 & 0,033 & 3,2 & $-0,7$ & 7,2 \\
\hline & Eq. PM16 & $14,8 \pm 5,4$ & 0,79 & 0,62 & 4,497 & 0,000 & 3,3 & $-1,5$ & 15,1 \\
\hline
\end{tabular}

Na FIGURA 1 são apresentadas dispersóes dos pontos relativos às diferenças médias (escores residuais) entre os resultados da $\mathrm{PH}$ e das equações empregadas neste estudo, de acordo com a plotagem de Bland e Altman ${ }^{16}$. Percebe-se a tendência de todas as equaçôes de Guedes e Guedes ${ }^{7}$ em superestimar o \%GC em relação à $\mathrm{PH}$. No entanto, em relação às equaçóes propostas por Petroski ${ }^{9}$, não existe um padrão, algumas superestimam e outras subestimam o \%GC. 

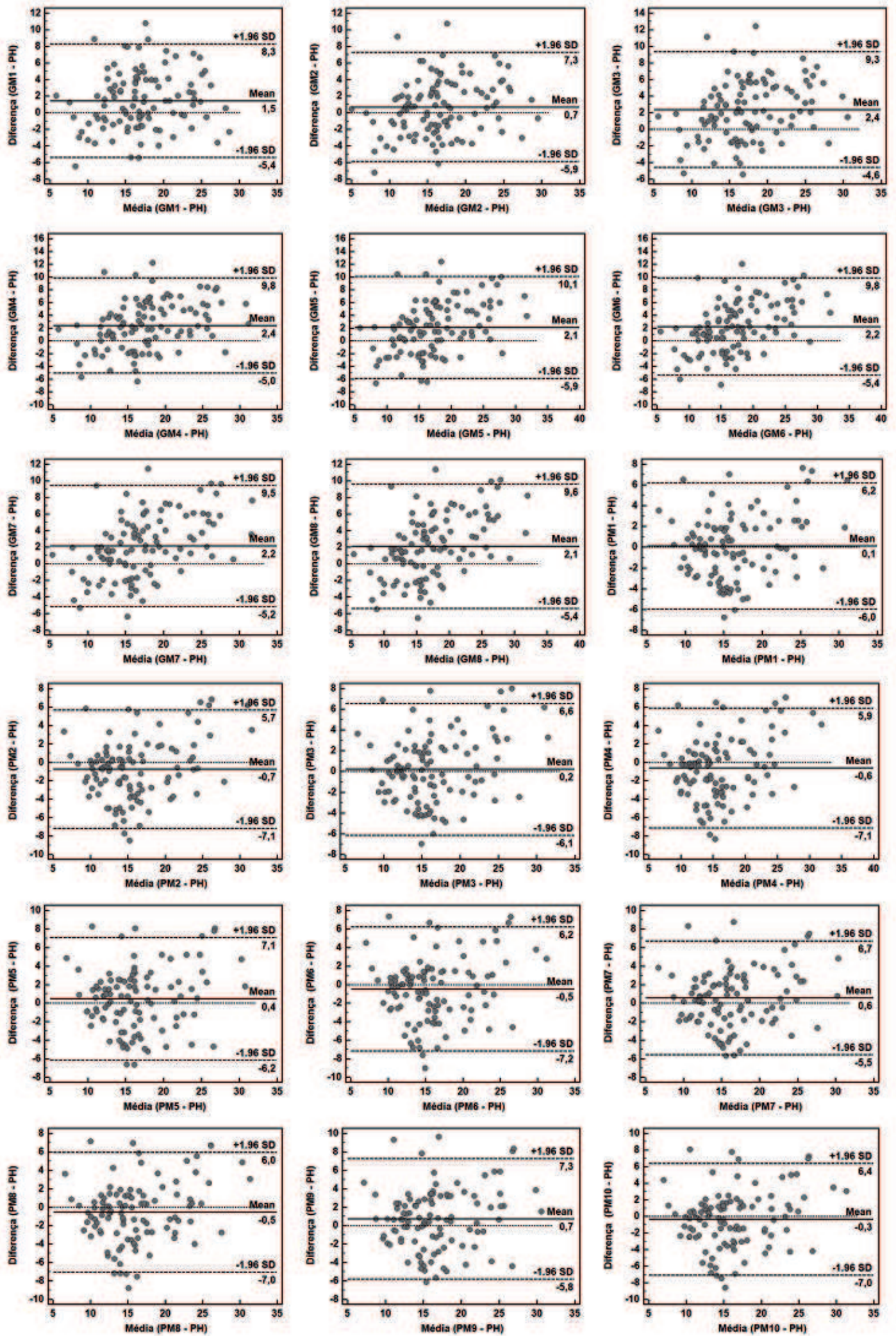

continua

FIGURA 1 - Gráficos de dispersão de BLAND e ALTMAN ${ }^{16}$ para comparações entre os valores de referência e os estimados pelas equações de Guedes e Guedes ${ }^{7}$ e de Petroski ${ }^{9}$. 

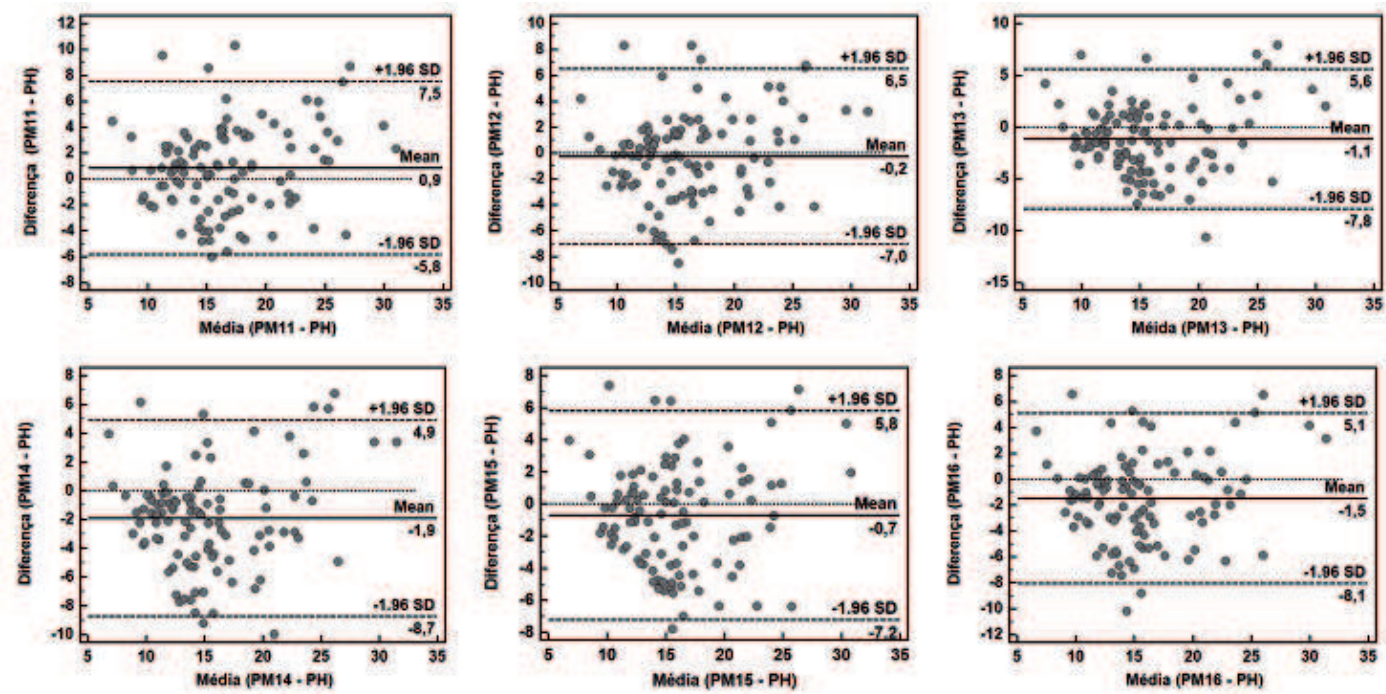

FIGURA 1 - Gráficos de dispersão de Bland e ALTMAN ${ }^{16}$ para comparações entre os valores de referência e os estimados pelas equações de Guedes e Guedes ${ }^{7}$ e de PETroski $^{9}$ (continuação).

\section{Discussão}

As equaçóes antropométricas antes de serem aplicadas a populaçóes diferentes da amostra de origem devem ser analisadas quanto a sua validade. Evidências apontam que a estimativa do \%GC pode diferir dependendo da escolha da equaçáo para estimar esta variável ${ }^{26}$.

No presente estudo, observou-se que as equaçóes testadas, em uma amostra de estudantes de Educação Física, apresentaram erros médios variando de $-1,9$ a 2,4 pontos percentuais em relação à medida adotada como critério $(\mathrm{PH})$.

Ao analisar as equaçóes desenvolvidas por GueDES e GuEdes ${ }^{7}$, era de se esperar que fornecessem os melhores resultados de validação, por serem específicas para a amostra utilizada neste estudo. No entanto, percebe-se que, de modo geral, não atenderam os critérios de validação cruzada. Apesar de apresentarem elevados valores de coeficiente de correlaçáo e determinação $\left(r \geq 0,77\right.$ e $r^{2} \geq 0,59$, respectivamente), foram evidenciadas diferenças significativas entre os resultados das equaçóes e os da PH. Ainda, os EPEs encontrados mostraram-se acima do recomendado, exceto para as equaçóes GM1 e GM2. O EPE fornece a variação padrão dos escores estimados em relaçáo à medida de referência. Esse cálculo, geralmente é utilizado na validação cruzada de equaçóes de estimativa da gordura corporal, considerando um EPE de 3,5\% como erro máximo a ser admitido ${ }^{25}$. O EC, que representa as diferenças entre os valores estimados e mensurados, demonstrou que todas as equaçóes de Guedes e Guedes $^{7}$ superestimaram os resultados de \%GC comparados aos da PH (TABELA 3).

Já o ET estabelece o erro associado ao número de indivíduos avaliados ${ }^{25}$ e também é empregado em estudos de validação. Quanto ao resultado desses cálculos, pode-se perceber que os menores valores foram observados para equaçóes que não apresentaram diferença estatisticamente significativa $(\mathrm{p}$ > 0,05) e com menores ECs.

Dentre as equaçōes de Guedes e Guedes ${ }^{7}$ a GM2 foi a que proporcionou os melhores resultados, com coeficiente de correlação de 0,81 , e de coeficiente de determinação de 0,66, EPE de 3,3\%, EC de 0,7\% e ET de 7,1\%, no entanto, apresentou diferença significativa quando comparada com a medida critério.

As equaçóes desenvolvidas por Petroski ${ }^{9}$ utilizam medidas de dobras cutâneas que variam de dois a nove pontos anatômicos, além dos perímetros do antebraço e do abdômen e a idade. Para essas equações, o EC variou de $-1,9 \%$ a $0,9 \%$ e os EPE apresentaram-se dentro do critério de validação $(\leq 3,5 \%)$ em comparação com a $\mathrm{PH}$. Quanto ao teste " $\mathrm{t}$ " para amostras dependentes, as equaçóes PM1, da PM3 a PM8, a PM10 e a PM12 não diferiram estatisticamente ( $\mathrm{p}$ > $0,05)$ do método critério. Os valores dos coeficientes de correlação e de determinação entre os métodos apresentaram-se elevados para todas as equações. 
Em uma investigação, $\mathrm{GoBBO}^{27}$ analisou a validade das equaçóes desenvolvidas por Petroski ${ }^{9}$ na estimativa do \%GC de universitários do sexo masculino, utilizando como método critério a DXA. Os resultados apontaram altos valores de correlaçáo $(r \geq 0,90)$ e EPE inferiores a 2,6\%, porém, todas as equaçóes de Petroski ${ }^{9}$ superestimaram o \%GC, apresentando diferença significativa.

Em outro estudo, FonseCA et al. ${ }^{13}$ investigaram a validade de equaçôes que estimam a Dc em 25 atletas profissionais de futebol, de 18 a 34 anos de idade. Entre as equaçôes analisadas, estavam as sugeridas por Petroski ${ }^{9}$ (Eq. PM7) e por Guedes e Guedes ${ }^{7}$ (Eq. GM3), no entanto, os pesquisadores não evidenciaram validade cruzada. Da mesma maneira, Moura et al. ${ }^{14}$ avaliaram a validade de várias equaçóes antropométricas na estimativa da composição corporal de 25 jogadores de futebol da categoria sub-20. Os autores utilizaram algumas equaçóes propostas por Petroski ${ }^{9}$ (Eq. PM8, PM10 e PM11) e as oito desenvolvidas por GUEDEs e Guedes ${ }^{7}$, porém, como demonstrado no estudo anterior, os resultados não apontaram validade para nenhuma equação.

Por outro lado, Glaner e Rodriguez-AŃEz ${ }^{15}$ em um estudo envolvendo 77 homens militares de Santa Maria - RS avaliaram, entre outras, a validade das 16 equaçóes propostas por Petroski ${ }^{9}$ e das oito desenvolvidas por Guedes e Guedes ${ }^{7}$, demonstrando que as equaçóes PM1 a PM4, PM6, PM8, PM10 e da PM12 a PM16 juntamente com a GM1 apresentaram validade cruzada.

Cabe ressaltar que os resultados distintos podem ter relação com as populações envolvidas nos estudos de Fonseca et al. ${ }^{13}$ e de Moura et al. ${ }^{14}$, os quais analisaram atletas de futebol.

Nesse sentido, observa-se que, antes de utilizar uma equação antropométrica indiscriminadamente, é necessário verificar se ela foi validada para a população a qual se deseja avaliar. Pois, como demonstrado nos estudos citados anteriormente, as equaçóes podem apresentar-se válidas ou não, dependendo da especificidade da população.

Considerando a análise dos escores residuais (FIGURA 1), cabe destacar que, tanto as pesquisas que deram origem as equações empregadas neste estudo, quanto a maioria das pesquisas que buscaram validar estas equaçôes, não empregaram a análise gráfica proposta por BLAND e ALTMAN ${ }^{16}$ para verificar o nível de concordância entre os métodos. Através desta análise, pode-se identificar que, quanto menor a dispersão, ou seja, menor o limite de concordância, que geralmente é um intervalo de confiança (IC) de $95 \%$, e o viés mais próximo de zero, mais concordantes são os resultados.

As equações de Guedes e GuEdEs ${ }^{7}$ produziram os maiores vieses, bem como as maiores dispersôes, com exceção da GM2. Já nas equaçôes desenvolvidas por Petroski ${ }^{9}$, foram observados os menores vieses, e baixos limites de concordância (FIGURA 1), indicando que essas equaçôes produzem resultados de $\% \mathrm{GC}$ mais precisos.

Considerando os critérios de validação adotados neste estudo, pode-se observar que a maioria das equaçóes generalizadas de Petroski ${ }^{9}$ apresentou os melhores resultados. As equaçóes PM1, da PM3 a PM8 e PM10 foram as que apresentaram validade cruzada. Entre os resultados das equaçóes antropométricas que cumpriram o estabelecido critério de validação, a PM1 destaca-se com os menores EC $(0,1 \%)$ e EPE $(3,1 \%)$; além disso, demonstrou o maior coeficiente de correlaçáo e determinação $(\mathrm{r}$ $\left.=0,84 ; r^{2}=0,70\right)$, bem como o menor limite de concordância (12,2\%), com IC de $-6,0 \%$ a 6,2\%.

Uma limitação deste estudo foi a utilização do adipômetro Cescorf (Cescorf Equipamentos Ltda.), não sendo de mesma marca que os utilizados nos estudos originais de Petroski ${ }^{9}$ e Guedes e Guedes ${ }^{7}$ no desenvolvimento de suas equaçóes, os quais utilizaram os da marca Lange ${ }^{\oplus}$ (Beta Technology Incorporated) e do tipo Harpenden ${ }^{\oplus}$ (British Indicators), respectivamente. No entanto, a literatura ainda é um pouco controversa quanto a possíveis interferências do tipo de compasso de dobras cutâneas nos resultados de composição corporal, pois assim como, CYrino et al. ${ }^{28}$ encontraram diferenças significativas nos resultados de \%GC utilizando os compassos Lange e Cescorf ${ }^{\bullet}$; Petroski et al. ${ }^{29}$ e SiLva et al. ${ }^{30}$ encontraram similaridade entre os resultados obtidos com estes mesmos adipômetros. Outra limitação importante foi a impossibilidade de mensurar diretamente o volume residual durante a pesagem subaquática, o que pode levar a erros na estimativa da gordura corporal.

De acordo com os resultados encontrados para a amostra estudada, pode-se concluir que entre as equações generalizadas desenvolvidas por Petroski ${ }^{9}$, oito apresentaram validade cruzada com o método critério (PM1, da PM3 a PM8 e a PM10). Dentre estas, a equação PM1 que utiliza como variáveis o somatório da espessura de nove dobras cutâneas e a idade, foi a que demonstrou a melhor concordância com o método de referência. Já, entre as equaçôes específicas desenvolvidas com universitários, propostas por GUEDES e GUEDEs ${ }^{7}$, nenhuma apresentou validade cruzada. 


\begin{abstract}
Validation of generalized and specific equations anthropometric to estimate the percentage of body fat in male Physical Education students

The objective of this study was to verify the validity of the specific equations GUEDES and GUEDES ${ }^{7}$ and generalized by PEtrosk ${ }^{9}$ in male college students. One hundred four students of Physical Education with a mean age of $21.7 \pm 2.7$ participated in the current study. They were submitted to the hydrostatic weighing (HW) and anthropometry (skinfolds and body circumferences). No statistically significant difference between measurements of fat percentage by $\mathrm{PH}$ and nine equations Petroskl ${ }^{9}$ was observed. All results obtained by equations GUEDES and GUEDES ${ }^{7}$ showed significant differences when compared with the PH. Among the equations analyzed eight developed by PETROSKI ${ }^{9}$ showed concurrent validity in the study sample.
\end{abstract}

KeY Words: Body composition; College students; Hydrostatic weighing; Anthropometry.

\title{
Referências
}

1. Cercato C, Mancini MC, Arguello AM, Passos VQ, Villares SM, Halpern A. Systemic hypertension, diabetes mellitus, and dyslipidemia in relation to body mass index: evaluation of a Brazilian population. Rev Hosp Clin Fac Med Sao Paulo. 2004;59:113-8.

2. Heyward V. ASEP methods recommendation: body composition assessment. J Exer Physiol. 2001;4:1-12.

3. Queiroga MR, Ferreira SA, Romanzini M. Perfil antropométrico de atletas de futsal feminino de alto nível competitivo conforme a função tática desempenhada no jogo. Rev Bras Cineantropom Desempenho Hum. 2005;7:30-4.

4. Salem M, Monteiro ABMC, Fernandes Filho J, Pires Neto CS. A composição corporal através da técnica da pesagem hidrostática. Rev Educ Fís. 2003;127:20-8.

5. Petroski EL, Pires-Neto CS. Validação de equaçóes antropométricas para a estimativa da densidade corporal em mulheres. Rev Bras Ativ Fís Saúde. 1995;1:65-73.

6. Slaughter MH, Lohman TG, Boileau RA, et al. Skinfold equations for estimation of body fatness in children and youth. Hum Biol. 1988;60:709-23.

7. Guedes DP, Guedes JERP. Proposição de equaçóes para a predição da quantidade de gordura corporal em adultos jovens. Semina. 1991;12:61-70.

8. Wilmore JH, Behnke AR. An anthropometric estimation of body density and lean body weight in young men. J Appl Physiol. 1969;27:25-31.

9. Petroski EL. Desenvolvimento e validação de equaçóes generalizadas para a estimativa da densidade corporal em adultos. Santa Maria: UFSM; 1995.

10. Visser M, van den Heuvel E, Deurenberg P. Prediction equations for the estimation of body composition in the elderly using anthropometric data. Br J Nutr. 1994;71:823-33.

11. Tran ZV, Weltman A. Generalized equation for predicting body density of women from girth measurements. Med SciSports Exerc. 1989;21:101-4.

12. Salem M, Fernandes Filho J, Pires Neto CS. Development and validation of specific anthropometric equations to determine the body density of Brazilian Army military women. Rev Bras Med Esporte. 2004;3:147-51.

13. Fonseca PHS, Marins JCB, Silva AT. Validação de equaçôes antropométricas que estimam a densidade corporal em atletas profissionais de futebol. Rev Bras Med Esporte. 2007;13:153-6.

14. Moura JAR, Rech CR, Fonseca PHS, Zinn JL. Validação de equaçôes para a estimativa da densidade corporal em atletas de futebol categoria sub-20. Rev Bras Cineantropom Desempenho Hum. 2003;5:22-32.

15. Glaner MF, Rodriguez-Añez CR. Validação de equaçóes para estimar a densidade corporal e/ou percentual de gordura para militares masculinos. Rev Treinamento Desport. 1999;4:29-36.

16. Bland J, Altman D. Statistical methods for assessing agreement between two methods of clinical measurements. The Lancet. 1986;12:307-10.

17. Atkinson G, Nevill AM. Statistical methods for assessing measurement error (reliability) in variables relevant to sports medicine. Sports Med. 1998;26:217-38. 
18. Barros MVG, Reis RS, Hallal PC, Florindo AA, Farias Júnior JC. Análise de dados em saúde. 3a ed. Londrina: Midiograf; 2012.

19. Ribeiro LG, Cardoso LO. Estudos de validação: qual análise utilizar? Rev Bras Med Esporte. 2009;15:216-7.

20. Stewart A, Marfell-Jones M, Olds T, Ridder Hd. International standards for anthropometric assessment. Lower Hutt: International Society for the Advancement of Kinanthropometry; 2011.

21. Petroski EL. Antropometria: técnicas e padronizaçôes. Jundiaí: Fontoura; 2011.

22. Heyward V. Avaliação física e prescrição de exercícios: técnicas avançadas. 4a ed. Porto Alegre: Artmed; 2004.

23. Goldman HI, Becklake MR. Respiratory function tests: normal values of medium altitudes and the prediction of normal results. Am Rev Respir Dis. 1959;79:457-67.

24. Heyward V, Wagner D. Applied body composition assessment. Champaign: Human Kinetics; 2004.

25. Lohman TG. Advances in body composition assessment. Champaign: Human Kinetics; 1992.

26. Rech CR, Lima LRA, Cordeiro BA, Petroski EL, Vasconcelos FAG. Validade de equaçôes antropométricas para a estimativa da gordura corporal em idosos do sul do Brasil. Rev Bras Cineantropom Desempenho Hum. 2010;12:1-7.

27. Gobbo LA. Validação de equaçóes antropométricas para a estimativa da massa muscular e da gordura corporal relativa a partir da absortometria radiológica de dupla energia em universitários do sexo masculino. Londrina: Universidade Estadual de Londrina; 2007.

28. Cyrino ES, Okano AH, Glaner MF, et al. Impacto da utilização de diferentes compassos de dobras cutâneas para a análise da composição corporal. Rev Bras Med Esporte. 2003;9:21-8.

29. Petroski EL, Velho NM, Pires-Neto CS. Validação do adipômetro Cescorf para estimar a composição corporal. I Jornada Integrada de Pesquisa Extensão e Ensino; Santa Maria, BR. Santa Maria: UFSM; 1994.

30. Silva IB, Seixas DW, Machado AF. Interferência da utilização de diferentes compassos de dobras cutâneas na avaliação da gordura corporal relativa. II Jornada de iniciação científica e tecnológica; São Paulo, BR. São Paulo; UNIBAN: 2008.

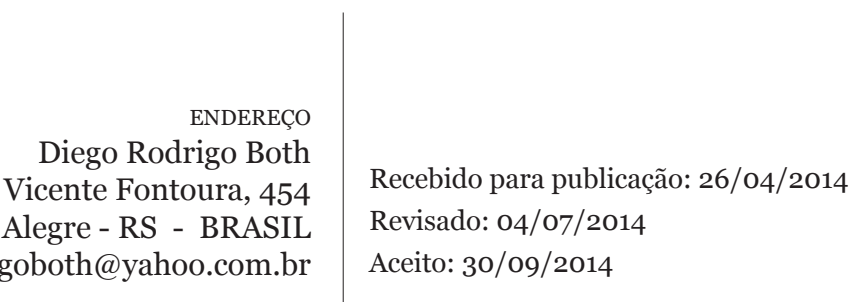

90640-00o - Porto Alegre - RS - BRASIL

e-mail: diegoboth@yahoo.com.br

Aceito: 30/09/2014 